\title{
Heavy flavour production at RHIC and LHC
}

\author{
Gian Michele Innocenti ${ }^{1, *}$ \\ ${ }^{1}$ Massachusetts Institute of Technology
}

\begin{abstract}
In this proceedings, I present selected experimental results on heavy-flavour production at RHIC and at the LHC, which were presented at the Strangeness in Quark Matter 2017 conference. I will present a brief introduction to the heavy-flavour physics in heavy ion collisions and I will focus on recents measurements of in-medium energy loss and and collective properties of heavy-flavour particles, which provided important information on the mechanisms of heavy flavour interaction with the hot and dense medium created in ultra-relativistic heavy-ion collisions.
\end{abstract}

\section{Introduction}

Heavy quarks are effective probes to study the properties of the deconfined medium created in heavy ion collisions ${ }^{1}$. These quarks, as a consequence of their large masses, are mostly produced in primary hard QCD scatterings with a production timescale that is shorter than the formation time of the QGP. During their propagation through the medium, heavy quarks lose energy via radiative and collisional interactions with the medium constituents. QCD predicts that quarks lose less energy than gluons as a consequence of their smaller colour factor. In addition, the so-called "dead-cone effect" is expected to reduce small-angle gluon radiation of heavy quarks when compared to both gluons and light quarks. Precise measurements of the nuclear modification factor $R_{\mathrm{AA}}$ of particles containing both light and heavy quarks can thus provide important tests for the predicted flavour-dependence of in-medium energy loss. In addition, the measurement of the $R_{\mathrm{AA}}$ of strange $\mathrm{D}$ mesons can also help estimating the relevance of the coalescence mechanism in which charmed hadrons are formed by the combination of charm quarks with light quarks from the medium. If coalescence plays a relevant role in the charm hadronisation, the yield of $\mathrm{D}_{\mathrm{s}}^{+}$particles are expected to be significantly enhanced with respect to the one of $\mathrm{D}^{0}$ mesons at low-intermediate transverse momenta $\left(\mathrm{p}_{\mathrm{T}}\right)$, as a consequence of the increased abundance of strange quarks in the deconfined medium. The production ratio of $\Lambda_{\mathrm{c}} / \mathrm{D}^{0}$ is also expected to be enhanced with respect to that of charmed mesons under the assumption that di-quarks bounded states in the quark-gluon plasma can be formed. Finally, the study of the collective properties of D and B mesons through detailed measurements of their azimuthal coefficienct $v_{n}$ at low-intermediate $\mathrm{p}_{\mathrm{T}}$ can also help quantifying the extent to which charm and beauty quarks flow with the medium, which is a good measure of their interaction strength and of their level of thermalisation.

The production of open heavy-flavours is measured at RHIC and at the LHC using

\footnotetext{
*e-mail: ginnocen@cern.ch

${ }^{1}$ More details and a complete bibliography for this section can be found in [1]
} 
several experimental techniques [1]. Charm and beauty hadrons can me measured via the exclusive reconstruction of their hadronic or semi-leptonic decay channels (e.g. $\mathrm{D}^{0} \rightarrow \mathrm{K}^{-} \pi^{+}$ or $\mathrm{B}^{+} \rightarrow \mathrm{J} / \Psi \mathrm{K}^{+} \rightarrow \mu^{+} \mu^{-} \mathrm{K}^{+}$) or via the inclusive reconstruction of semi-leptonic decays (e.g. $\mathrm{B} \rightarrow \mathrm{J} / \Psi+\mathrm{X}$ ). The production of heavy-flavour particles can also be studied up to very high $\mathrm{p}_{\mathrm{T}}$ by measuring jets of hadrons produced in the fragmentation of a charm or beauty quark. Charm and beauty decays are characterised by relativetely small cross sections and by decay vertices which are displaced few hundreds $\mu \mathrm{m}$ from the main interaction vertices. Therefore the study of these observables requires very large statistics of minimum-bias and triggered events and precise tracking and vertexing detectors that can operate under conditions of very large detector occupancy.

\section{Proton-proton measurements}

The study of heavy-flavour production in proton-proton collisions is considered a fundamental reference for nucleus-nucleus measurements but at the same time it provides an important benchmark for perturbative QCD calculations based on a factorisation approach [1]. The possibility of measuring the charm and beauty production both at RHIC and the LHC is crucial to constrain the relative relevance of different production mechanisms (e.g. flavour creation vs gluon fusion processes), which are expected to play a different role at different energies. The $\mathrm{p}_{\mathrm{T}^{-}}$and $y$-differential cross sections of charm and beauty quarks were measured by several collaborations both at RHIC and at the LHC [2-13] and were found to be consistent with perturbative QCD calculations (e.g. FONLL[16]) that include a consistent description of next-to-leading order processes. As an example, in the left panel of Fig. 1, the $\mathrm{D}^{0}$ production cross sections as a function of $\mathrm{p}_{\mathrm{T}}$ measured by the ALICE experiment at $\mathrm{LHC}$ at $7 \mathrm{TeV}$ and by the STAR experiment at RHIC at 200 and $500 \mathrm{GeV}$ are presented. In the central panel of the same figure, the $\mathrm{B}^{+}$production measured by the ATLAS experiment at $7 \mathrm{TeV}$ is shown. Although theoretical calculations are able to describe successfully single-differential observables, more differential measurements like DD or $\mathrm{B} \overline{\mathrm{B}}$ azimuthal correlations [14, 15] showed that the current understanding of next-to-leading order processes at least at the LHC is still not satisfactory. In particular, the contribution of the gluon-splitting production mechanism $(g \rightarrow c \bar{c}$ or $g \rightarrow b \bar{b})$, which is dominant at small angles between the particle-anti-particle pairs is sizeably understimated by theoretical calculations (right panel of Fig. 1). A better description of the gluon splitting processes would be extremely important since charm and beauty quarks produced via these mechanisms are mostly sensitive to the energy loss of gluons instead of that of the heavy parton.

\section{Proton-nucleus measurements}

In presence of a nuclear environment, the yield of production of light and heavy particles can be modified in absence of any deconfined medium. In particular, modifications of the parton distribution functions (PDFs) in the nucleus with respect to nucleon PDFs (e.g. due to shadowing) are expected to be responsable for sizeable modifications of the heavy-flavour cross section at low transverse momenta [1]. In order to quantify the effects of these phenomena and validate the hypothesis that the suppression observed in nucleus-nucleus collisions is a consequence of the presence of a a hot and dense medium, one can study the heavy-flavour production in proton-nucleus collisions, where the formation of the Quark-Gluon Plasma is not expected. In the last years, the role of "reference" of proton-nucleus measurements have been deeply questioned. However, at the present moment, no measurement has highlighted the presence of sizeable jet quenching phenomena in proton-nucleus or proton-proton collisions, suggesting that measurements of production yields in smaller collision systems can still 

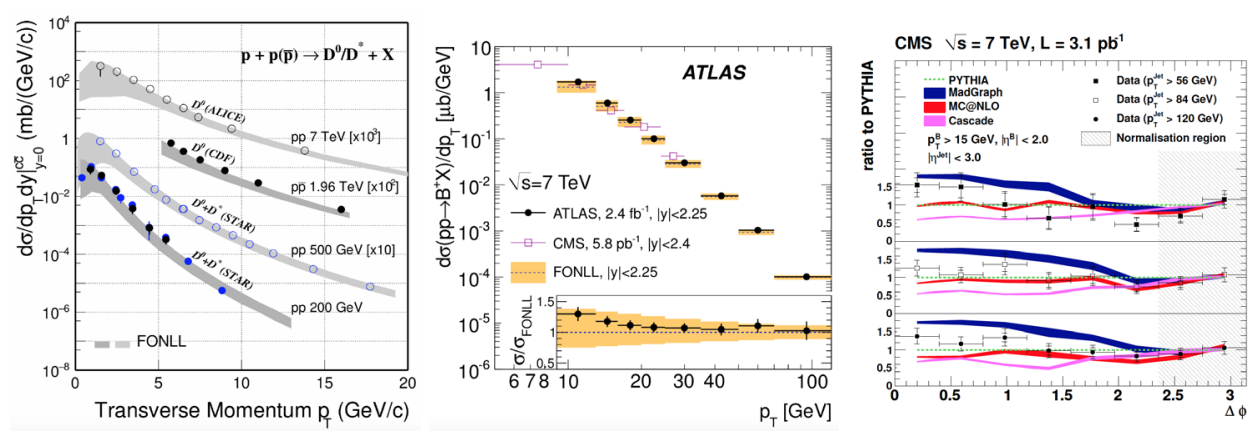

Figure 1. (Left) $\mathrm{D}^{0}$ production cross sections measured by the ALICE experiment at LHC in pp collisions at $7 \mathrm{TeV}$ and by the STAR experiment at RHIC at 200 and $500 \mathrm{GeV}$. (Middle) $\mathrm{B}^{+}$production measured by the ATLAS experiment in pp collisions at $7 \mathrm{TeV}$. (Right) $\mathrm{B} \overline{\mathrm{B}}$ azimuthal correlation measured by the CMS experiment in pp collisions at $7 \mathrm{TeV}$.

be used to isolate the effect of cold nuclear matter effects. The nuclear modification factors $R_{p \mathrm{~A}}$ of heavy-flavoured particles have been measured both at RHIC and at the LHC [17-23]. Thanks to the very high statistics $2016 \mathrm{pPb}$ run, the $R_{p \mathrm{~A}}$ of $\mathrm{D}^{0}$ mesons have been measured at the LHC with very high precision (Fig. 2). The measurements performed by LHCb at forward and backword rapidity have highlighted for the first time significant modifications of the production yields of $\mathrm{D}$ mesons for $\mathrm{p}_{\mathrm{T}}<10 \mathrm{GeV}$ in $\mathrm{pPb}$ collisions, which are compatible with the predictions of models that include the effect of modifications of nuclear PDFs. The measurement performed by ALICE at central rapidity and an a simple rapidity extrapolation of the $\mathrm{LHCb}$ measurement show that at central rapidity the effect of cold nuclar matter effects at low $\mathrm{p}_{\mathrm{T}}$ is smaller or equal to about $15 \%$. At higher $\mathrm{p}_{\mathrm{T}}$, as expected, no significant deviations from binary scaling $\left(R_{p \mathrm{~A}}=1\right)$ was observed. In the beauty sector at the LHC, measurements of heavy-flavour electrons performed by ALICE and exclusive B-meson measurements from CMS have measured $R_{p \mathrm{~A}}$ consistent with unity from 1 to $60 \mathrm{GeV} / \mathrm{c}$ at mid rapidity with uncertainties of about 30-40\%. A recent CMS measurement of the non-prompt $J / \Psi R_{p \mathrm{~A}}$ has measured at central rapidity $R_{p \mathrm{~A}}=1$ with uncertainty smaller than $15 \%$. A new impressive LHCb measurement of non-prompt $J / \Psi R_{p \mathrm{~A}}$ performed down to $0 \mathrm{GeV} / \mathrm{c}$ has highlighted for the first time a significant deviation from unity for the $R_{p \mathrm{~A}}$ at forward rapidity as expected in presence of nuclear shadowing also in the beauty sector. Also at the RHIC energies, the $R_{p \mathrm{~A}}$ of beauty has been recently measured via the reconstruction of non-prompt $J / \Psi$ decays. The PHENIX collaboration measured a $R_{p \mathrm{~A}}$ consistent with unity with uncertainties of the order of $40-50 \%$.

\section{Nucleus-nucleus measurements}

Indications of a strong charm suppression at the LHC are observed by the ALICE and the CMS collaborations using the $R_{\mathrm{AA}}$ of promptly produced $\mathrm{D}$ mesons at mid-rapidity at a nucleon-nucleon centre-of-mass energy $\sqrt{s_{\mathrm{NN}}}=2.76$ and $\sqrt{s_{\mathrm{NN}}}=5.02 \mathrm{TeV}$ [24-26]. The results of the two collaborations are fully consistent if compared in the same $\mathrm{p}_{\mathrm{T}}$ and centrality region (left and middle panel of Fig. 3). The $R_{\mathrm{AA}}$ of $\mathrm{D}^{0}$ mesons at the LHC shows a maximum suppression of a factor 5-6 with respect to the pp reference for the $10 \%$ most central collisions in the $\mathrm{p}_{\mathrm{T}}$ range of $6-10 \mathrm{GeV} / \mathrm{c}$. For $\mathrm{D}^{0}$ mesons in the high- $\mathrm{p}_{\mathrm{T}}$ range of

\footnotetext{
${ }^{1}$ Biased opionion of the author.
} 

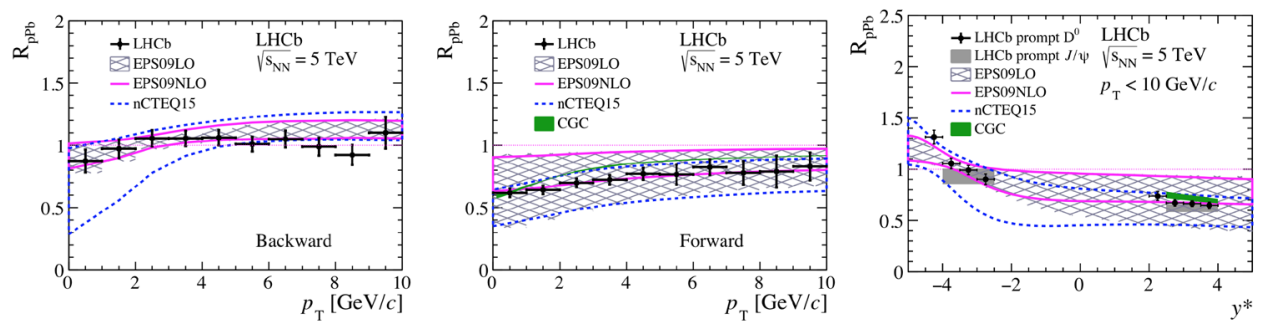

Figure 2. $\mathrm{D}^{0} R_{p \mathrm{~A}}$ in the backward (left) and forward (right) rapidity as a function of $\mathrm{p}_{\mathrm{T}}$ and as a function of rapidity for $\mathrm{p}_{\mathrm{T}}<10 \mathrm{GeV} / \mathrm{c}$ measured by the $\mathrm{LHCb}$ collaboration in pp collisions at $7 \mathrm{TeV}$.

$60-100 \mathrm{GeV} / \mathrm{c}$, a significantly smaller suppression is observed. The $R_{\mathrm{AA}}$ as a function of $\mathrm{p}_{\mathrm{T}}$ is quite well described by several theoretical calculations that include collisional or radiative energy loss or a mixture of the two mechanisms, confirming that the $R_{\mathrm{AA}}$ alone is not powerful enough to discriminate between models even if measured in a very wide kinematic range. An increasing suppression is observed as a function of centrality as expected in case of the creation of a denser and hotter medium in central $\mathrm{PbPb}$ collisions. This trend is also well described by theoretical calculations. Quite surprisingly, at the LHC the $R_{\mathrm{AA}}$ of $\mathrm{D}^{0}$ mesons was found to be almost completely consistent with the charged particle $R_{\mathrm{AA}}$ from 1 to 100 $\mathrm{GeV} / \mathrm{c}$ [32]. The same $R_{\mathrm{AA}}$, however, in presence of very different $\mathrm{p}_{\mathrm{T}}$-spectra and fragmentation functions does not imply the same energy loss. On the contrary, the evidence of a very similar suppression for charged particles and D mesons seems to be a very fortunate (too fortunate?) cancellation of the effects of different energy loss, $\mathrm{p}_{\mathrm{T}}$-spectra and fragmentation functions. Although described by several theoretical calculations, this observation does not present a clear and unique physics interpretation ${ }^{1}$. At RHIC energies, the $\mathrm{D}^{0} R_{\mathrm{AA}}$ was also measured by the STAR Collaboration. The D-meson $R_{\mathrm{AA}}$ measured at $200 \mathrm{GeV}$ presents values larger than 1.5 even if with large uncertaintes at $\mathrm{p}_{\mathrm{T}}<2 \mathrm{GeV} / \mathrm{c}$, being significantly higher than the charged particle $R_{\mathrm{AA}}$ in the same transverse momentum region (right panel of Fig. 3 ). Such a difference between the ratio of the light and D-meson $R_{\mathrm{AA}}$ at the two energies might be attributed to differences in radial flow or nuclear PDF modifications at the two energies.
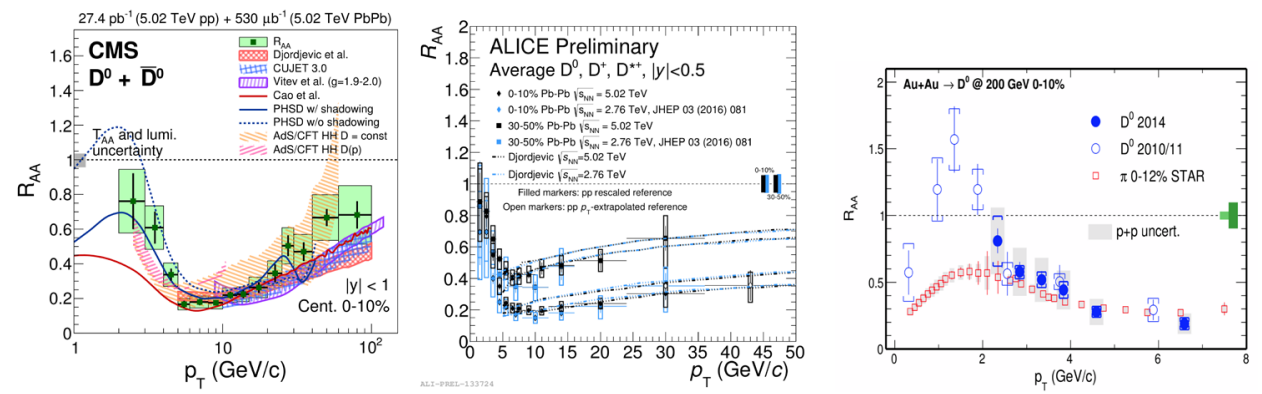

Figure 3. (Left) $\mathrm{D}^{0}$ nuclear modification factor for central $\mathrm{PbPb}$ collisions at $5.02 \mathrm{TeV}$ measured by CMS. (Center) $\mathrm{D}^{0}$ nuclear modification factor for central and semi-peripheral collisions measured by ALICE. (Right) $\mathrm{D}^{0}$ nuclear modification factor for central AuAu collisions at $200 \mathrm{GeV}$ measured by STAR compared to the one of charged particles in a similar centrality range. 
The suppression of beauty particles was also measured by different collaborations both at RHIC and at the LHC [27-31]. At the LHC, the $R_{\mathrm{AA}}$ of beauty particles was measured down to $2 \mathrm{GeV}$ by the ALICE experiment with heavy-flavour electron measurements and from 3 $\mathrm{GeV}$ up to $50 \mathrm{GeV}$ using exclusively reconstructed $\mathrm{B}$ mesons and non-prompt $\mathrm{B}$ meson decays $(\mathrm{B} \rightarrow \mathrm{J} / \Psi+\mathrm{X})$. As shown in the left and middle panel Fig. 4, the current measurements indicate a hint of different suppression of beauty and charmed particles at low-intermediate $\mathrm{p}_{\mathrm{T}}$ that vanishes when going to higher transverse momenta. Several reasonable concerns were raised with respect to the CMS comparison plot. The first one is related to the fact that in this plot the $\mathrm{p}_{\mathrm{T}}$ of the non-prompt $\mathrm{J} / \Psi$ is not corrected for the difference between the $\mathrm{p}_{\mathrm{T}}$ of the beauty hadrons and the $\mathrm{p}_{\mathrm{T}}$ of the non-prompt $\mathrm{J} / \Psi$. This is a valid point that should be addressed in future version of this result expecially for what concerns the low $\mathrm{p}_{\mathrm{T}}$ region. At higher $\mathrm{p}_{\mathrm{T}}$, the fact that the $R_{\mathrm{AA}}$ does not show a strong $\mathrm{p}_{\mathrm{T}}$-dependence should make this missing correction less relevant. The second concern is related to the possible discrepancy between the non-prompt $\mathrm{J} / \Psi$ and the exclusive $\mathrm{B}^{+}$meson result. In this case, I would like to stress the fact that the uncertainties on the current $\mathrm{B}^{+}$meson $R_{\mathrm{AA}}$ are quite sizeable and the two measurements are still compatible within the uncertainties. In addition, it has to be noticed that in principle the non-prompt $\mathrm{J} / \Psi$ result might be affected by effects of enhancement of strange beauty mesons and baryons that could be not completely negligible in this $\mathrm{p}_{\mathrm{T}}$ range. The last concerns is related to the fact that the results reported here mix measurements performed in different rapidity regions. To addess this point, more differential analysis of the $R_{\mathrm{AA}}$ of the different species as a function of rapidity should be performed ${ }^{2}$. However, the recent measurement of non-prompt $\mathrm{J} / \Psi$ of $\mathrm{CMS}$ as function of the rapidity does not indicate a strong $\mathrm{y}$-dependence of the suppression. This evidence seems to indicate that the different rapidity coverage of the measurements should not affect the physics conclusions we draw with the current version of this plot. At RHIC the suppression of beauty hadrons was also measured by the STAR and the PHENIX collaborations using non-prompt $\mathrm{J} / \Psi$ and heavyflavour electrons. Also at this energies, as shown in the right panel Fig. 4 in the PHENIX heavy-flavour electron results, a hint of different suppression is observed for $\mathrm{p}_{\mathrm{T}}$ smaller than about $6 \mathrm{GeV} / \mathrm{c}$.
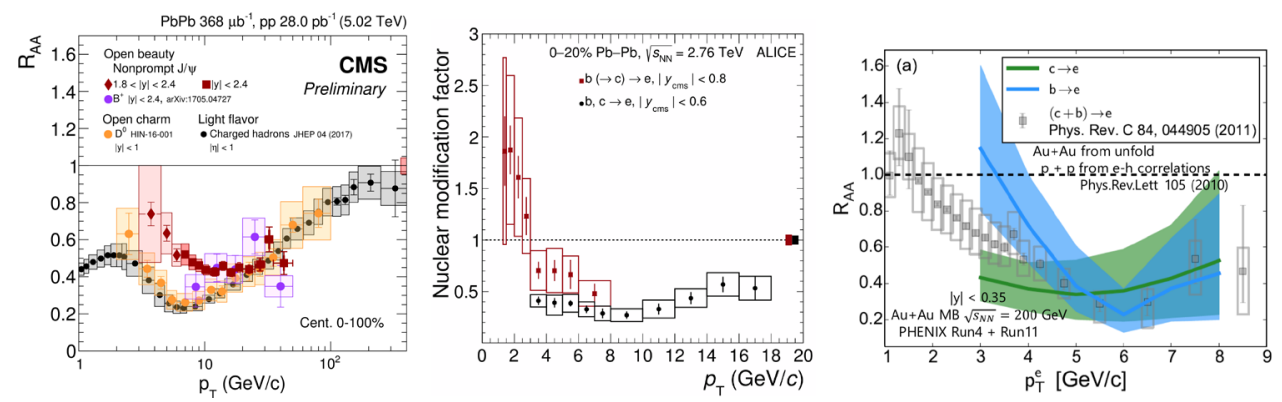

Figure 4. (Left) Nuclear modification factor of charged particles, $\mathrm{D}^{0}$ mesons, non-prompt $\mathrm{J} / \Psi$ and $\mathrm{B}^{+}$ mesons performed by $\mathrm{CMS}$ at in $\mathrm{PbPb}$ collisions at $5.02 \mathrm{TeV}$. (Center) Nuclear modification factor of heavy flavoured electrons performed by ALICE in central $\mathrm{PbPb}$ collisions at $5.02 \mathrm{TeV}$. (Right) Nuclear modification factor of heavy flavoured electrons performed by PHENIX in minimum-bias $\mathrm{PbPb}$ collisions at $200 \mathrm{GeV}$.

\footnotetext{
${ }^{2}$ The reason why this was not done yet is related to the fact that the a B-meson measurement in a smaller rapidity region is currently not possible due to the limited statistics and the non-prompt $\mathrm{J} / \Psi$ measurement at central rapidity cannot reach the very low-momentum region as a consequence of the kinematic cuts applied to the muons and to the characteristics of the CMS muon detectors.
} 
A first indication of a relevant contribution of recombination in the open charm sector was recently observed both at RHIC and at the LHC [33, 34]. A hint of a larger $R_{\mathrm{AA}}$ of $\mathrm{D}_{\mathrm{S}}^{+}$ mesons with respect to non-strange D-meson one was observed by the ALICE and the STAR collaboration in central and semi-central collisions in the low-intermediate $\mathrm{p}_{\mathrm{T}}$ region. This is currently interpretated as an indication that coalescence is playing a relevant role in the hadronisazion of charm quarks in heavy-ion collisions. The observation of this phenomenon seems to confirm the presence of free color charges in the medium. In absence of color connectivity indeed, recombination processes cannot take place. A precise estimation of the effect of recombination is also very important as an input for theoretical calculations that describe the suppression of heavy-flavoured particles. A wrong estimation of recombination effects can indeed bias our understanding of the magnitude of energy loss processes. The STAR Collaboration also recently reported a very interesting first measurement of the $\Lambda_{\mathrm{c}}$ $R_{\mathrm{AA}}$ in semi-peripheral gold-gold collisions. Also in this case, an ehnancement with respect to the pp baseline was observed. This result can be intepreted theoretically if we assume that charm recombines in a medium where di-quark bound states are formed.
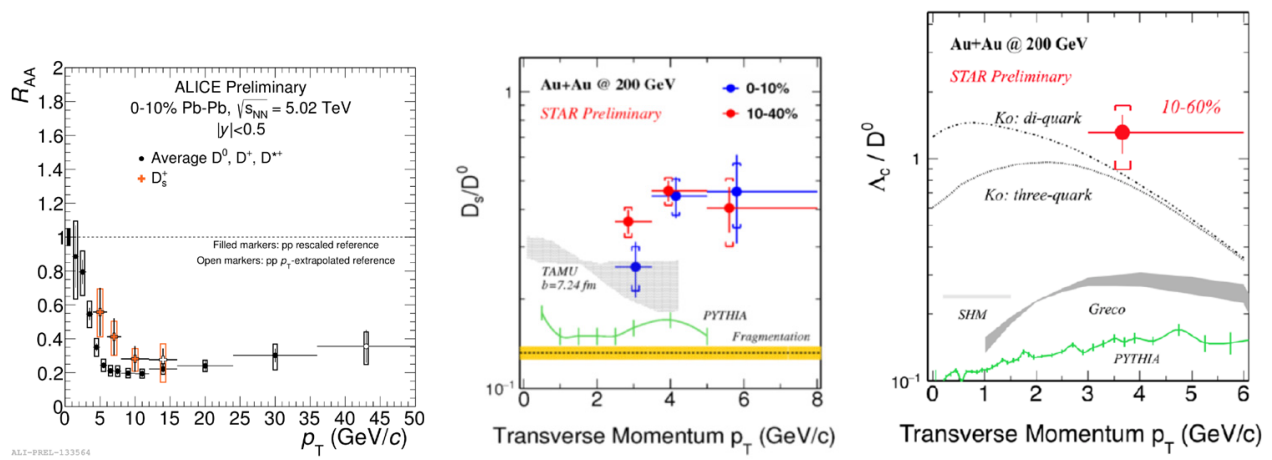

Figure 5. (Left) Nuclear modification factor of $\mathrm{D}_{\mathrm{s}}^{+}$mesons performed by ALICE in $\mathrm{PbPb}$ collisions at $5.02 \mathrm{TeV}$ compared to the result for non-strange $\mathrm{D}$ mesons. (Center) $\mathrm{D}_{\mathrm{s}}^{+} / \mathrm{D}^{0}$ meson ratio performed by STAR in central and semi-central AuAu collisions at $200 \mathrm{GeV}$. (Right) $\Lambda_{c} / \mathrm{D}^{0}$ meson ratio performed by STAR in AuAu collisions at $200 \mathrm{GeV}$ in the centrality range $0-80 \%$.

Strong indications of charm flow were also observed by several collaborations both at RHIC and at the LHC [35-37]. Large and positive values of the elliptic flow coefficients $v_{2}$ were measured by ALICE and CMS at low-intermediate $\mathrm{p}_{\mathrm{T}}$ in semi-pheripheral $\mathrm{PbPb}$ collisions and by STAR in AuAu collisions (left and middle panel of Fig. 6). Due to the limited statistics available, the STAR result was performed in an integrated centrality interval 0 $80 \%$. Therefore a direct comparison between the magnitude of the elliptic flow at the two energies cannot be currently done. At the LHC, where more differential measurement were performed, the magnitude of the D-meson $v_{2}$ coefficient was found to decrease when going from peripheral to more central events as expected in case the elliptic flow is generated as a consequence of the initial anysotropy of the fireball. A non-zero $v_{2}$ is also observed at larger $\mathrm{p}_{\mathrm{T}}(>10 \mathrm{GeV} / \mathrm{c})$. In this kinematic region, the $v_{2}$ measurement is no longer sensitive to collective phenomena but it is sensitive to the in-medium path dependence of the energy loss and can be therefore use to contraint jet quenching theoretical calculations. At the LHC, a significant indication of smaller $v_{2}$ of $\mathrm{D}$ mesons with respect to charged-particle $v_{2}$ was observed and a first hint of non-zero $v_{3}$ of D mesons was also measured by CMS in non-central collisions. These high-precision results provided strong constraints on theoretical calcula- 
tions. An important theoretical effort is ongoing to use these measurements to quantify the level of charm thermalisation in the medium, to understand if charm quarks can be described by a hydro-dynamic description and to extract the difficusion parameters of charm quarks in the medium. The shape of the $v_{2} \mathrm{D}$-meson measurement, in addition, can provide important information on the relative relevance of collisional and radiative energy loss processes, being therefore complementare to the the $R_{\mathrm{AA}}$ measurement. At the LHC, a first attempt was also made by the CMS collaboration to measure the $v_{2}$ coefficient of beauty by the measurement of non-prompt $\mathrm{J} / \Psi$ in semi-peripheral $\mathrm{PbPb}$ events. The current result (right panel of Fig. 6), even if still not conclusive, represents a very promising attempt to estimate the collective behavior of beauty charms in heavy-ion collisions.
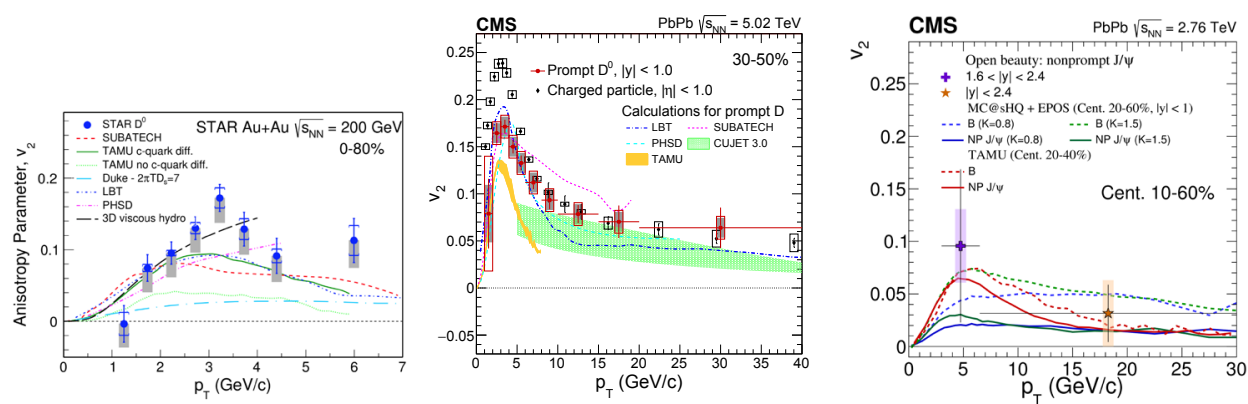

Figure 6. (Left) $v_{2}$ coefficient of $\mathrm{D}^{0}$ mesons performed by STAR in AuAu collisions at $200 \mathrm{GeV}$ in the centrality range $0-80 \%$. (Center) $v_{2}$ coefficient of $\mathrm{D}^{0}$ mesons performed by CMS in semi-peripheral $\mathrm{PbPb}$ collisions at $5.02 \mathrm{TeV}$. (Right) $v_{2}$ coefficient of non-prompt $\mathrm{J} / \Psi$ performed by CMS in semiperipheral $\mathrm{PbPb}$ collisions at $5.02 \mathrm{TeV}$.

\section{Conclusions}

In the last few years, impressive results were obtained in the sector of heavy-flavour observables in heavy-ion collisions, moving our field to an exciting era of high-precision measurements. Although a first quantitative understanding of heavy-flavour phenomena was achieved, a long way is still ahead in order to be able to use heavy-flavour observables to improve our understanding of the the properties of the Quark-Gluon Plasma. In the coming future, the new high-luminosity runs at RHIC and LHC and the upgraded detectors will allow us to improve the precision of the traditional observables but also to perform new more differential measurements (like D-hadron, D-jet, D-D̄ correlations, D-meson event-shape engineering measurements) that can contraint better the mechanisms of heavy-flavour production and the mechanisms of in-medium energy loss. A better and quantitative understanding of the microscopic mechanisms of heavy-quark interaction with the medium (energy loss mechanisms and hadronisation mechanisms in particular) is a pre-requisite in order to use theoretical calculations to extract fundamental properties like the transport coefficients or the the $\hat{q}$ coefficient. At the same time, measurements of heavy-flavour productions in smaller systems should be exploited to better understand the properties and the nature of the collective phenomena that were recently observed in both pp and proton-nucleus collisions.

\section{References}

[1] A. Andronic et al., Eur. Phys. J. C 76 (2016) 107, doi:10.1140/epjc/s10052-015-3819-5, arXiv:1506.03981. 
[2] STAR Collaboration, Phys.Rev.D86(2012)072013.

[3] CDF Collaboration,etal.,Phys.Rev.Lett.91(2003)241804.

[4] ALICE Collaboration, et al., JHEP 01 (2012) 128.

[5] ALICE Collaboration, et al., arXiv:1702.00766.

[6] CMS Collaboration, Phys.Rev.Lett.106(2011)112001.

[7] ATLAS Collaboration, JHEP 10 (2013) 042.

[8] CMS Collaboration, et al., arXiv:1609.00873.

[9] C. Aidala, et al., arXiv:1701.01342.

[10] LHCb Collaboration, JHEP 03 (2016) 159.

[11] LHCb Collaboration, JHEP 08 (2013) 117.

[12] PHENIX Collaboration, arXiv:1405.3301

[13] LHCb Collaboration, JHEP 1603 (2016) 159, Erratum: JHEP 1705 (2017) 074.

[14] CMS Collaboration, JHEP03(2011)136.

[15] LHCb Collaboration, JHEP06(2012)141.

[16] M. Cacciari,P.Nason,R.Vogt,Phys.Rev.Lett.95(2005)122001.

[17] ALICE Collaboration, Phys.Rev.Lett.113(23)(2014)232301.

[18] ALICE Collaboration,ALICE-PUB-2017-008.

[19] LHCb Collaboration, arXiv:1707.02750v1.

[20] ALICE Collaboration, EPJC 77 (2017) 269.

[21] ALICE Collaboration, arXiv:1609.03898.

[22] LHCb Collaboration, these proceedings.

[23] PHENIX Collaboration, arXiv.1702.01085.

[24] CMS Collaboration, arXiv:1708.04962v1.

[25] ALICE Collaboration, ALICE-PUBLIC-2017-003.

[26] STAR Collaboration, PRL118, 212301.

[27] CMS Collaboration, arXiv 1705.04727,

[28] CMS Collaboration, arXiv: 1610.00613 EPJC 77 (2017) 252.

[29] ALICE Collaboration, arXiv:1609.03898.

[30] STAR Collaboration, these proceedings.

[31] PHENIX Collaboration, these proceedings.

[32] CMS Collaboration, arXiv: 1611.01664 JHEP 04 (2017) 039.

[33] ALICE Collaboration, ALICE-PUBLIC-2017-003.

[34] STAR Collaboration, these proceedings.

[35] CMS Collaboration, arXiv:1708.03497v1.

[36] ALICE Collaboration, arXiv:1707.01005v1.

[37] STAR Collaboration, PRL118, 212301. 